Note: This is a draft of a paper submitted for publication. Contents of this paper should not be quoted or referred to without permission of the author(s).

To be presented at Fall Meeting of the Materials Research Society,

Boston, MA, November 28-December 28, 1994 and published in Beam-Solid Interactions for Materials Synthesis and Characterization, ed. by D. E. Luzzi, T. F. Heinz, M. Iwaki, and D. C. Jacobson, Materials Research Society, Pittsburgh, PA DYNAMIC DEPOSITION PROCESSES FOR PULSED-LASER DEPOSITION OF THIN FILMS

C. L. Liu, J. N. LeBoeuf, R. F. Wood, D. B. Geohegan, J. M. Donato, K. R. Chen, and A. A. Puretzky

Solid State Division, Oak Ridge National Laboratory

P.O. Box 2008, Oak Ridge, Tennessee 37831-6030

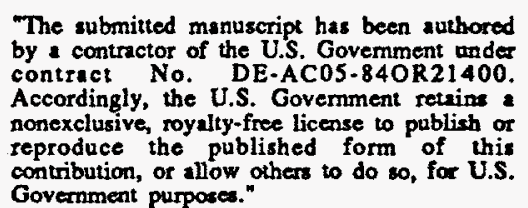

Government purposes."

SOLID STATE DIVISION

OAK RIDGE NATIONAL LABORATORY

Managed by

MARTIN MARIETTA ENERGY SYSTEMS, INC.

under

Contract No. DE-AC05-84OR21400

with the

U.S. DEPARTMENT OF ENERGY

Oak Ridge, Tennessee

November 1994

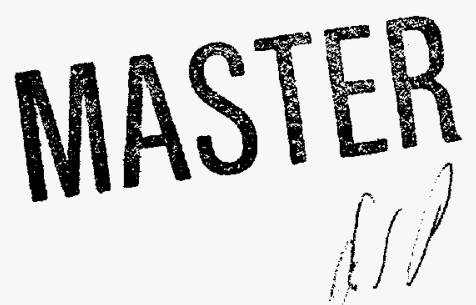




\section{DISCLAIMER}

This report was prepared as an account of work sponsored by an agency of the United States Government. Neither the United States Government nor any agency thereof, nor any of their employees, make any warranty, express or implied, or assumes any legal liability or responsibility for the accuracy, completeness, or usefulness of any information, apparatus, product, or process disclosed, or represents that its use would not infringe privately owned rights. Reference herein to any specific commercial product, process, or service by trade name, trademark, manufacturer, or otherwise does not necessarily constitute or imply its endorsement, recommendation, or favoring by the United States Government or any agency thereof. The views and opinions of authors expressed herein do not necessarily state or reflect those of the United States Government or any agency thereof. 


\section{DISCLAIMER}

Portions of this document may be illegible in electronic image products. Images are produced from the best available original document. 


\title{
MODELING OF THERMAL, ELECTRONIC, HYDRODYNAMIC, AND DYNAMIC DEPOSITION PROCESSES FOR PULSED-LASER DEPOSITION OF THIN FILMS
}

\author{
C. L. Liu, J. N. Leboeuf,* R. F. Wood, D. B. Geohegan, \\ J. M. Donato,** K. R. Chen, ${ }^{*}$ and A. A. Puretzky \\ Solid State Division, Oak Ridge National Laboratory \\ Oak Ridge, TN 37831-6032 \\ *Fusion Energy Division, ORNL \\ **Engineering Physics and Mathematics Division, ORNL
}

\begin{abstract}
Various physical processes during laser ablation of solids for pulsed-laser deposition (PLD) are studied using a variety of computational techniques. In the course of our combined theoretical and experimental effort, we have been trying to work on as many aspects of PLD processes as possible, but with special focus on the following areas: (a) the effects of collisional interactions between the particles in the plume and in the background on the evolving flow field and on thin film growth, (b) interactions between the energetic particles and the growing thin films and their effects on film quality, (c) rapid phase transformations through the liquid and vapor phases under possibly nonequilibrium thermodynamic conditions induced by laser-solid interactions, (d) breakdown of the vapor into a plasma in the early stages of ablation through both electronic and photoionization processes, (c) hydrodynamic behavior of the vapor/plasma during and after ablation. The computational techniques used include finite difference (FD) methods, particle-in-cell model, and atomistic simulations using molecular dynamics (MD) techniques.
\end{abstract}

\section{Introduction}

One of the main techniques for laser materials processing is pulsed laser deposition for thin film growth. The advantages of PLD compared to other techniques include, novel epitaxial and low temperature growth of homogeneous and heterogeneous films by utilizing energetic species, stoichiometric ablation of constituent species of the target, and growth of metastable phases layer-by-layer under nonequilibrium ablation conditions. However, there are still several issues, such as inclusion of particulates in the growing films and spatial non-uniformities of the films over large areas, that must be resolved before PLD can be widely used for industrial applications. While experimentalists are trying to find optimal conditions for thin film growth by PLD, a systematic effort in modeling of various physical processes during deposition is needed. In this paper, we present preliminary results from theoretical modeling of laser-ablation phenomena using a variety of computational techniques. For computational modeling of the complicated processes such as occur during PLD, one faces the challenge not only to better understand the fundamentals of the processes, but also to utilize the most appropriate computational techniques in the modeling.

\section{Pulsed laser melting and evaporation}

A variety of phenomena and issues, such as melting, solidification, overheating and undercooling, nucleation, coexisting multiple phases, rapidly moving multiple phase boundaries, interfacial kinetics, temperature/phase-dependent optical and thermal properties of the materials, etc., have to be dealt with in order to properly model laser-solid interactions. In the LASER8 programs of 1-D [1,2] and 2-D [3,4] models, finite difference (FD) methods were used to solve heat flow equations in terms of enthalpy and temperature. A concept of state arrays was developed to monitor phase status and other parameters in each finite difference cell. These 
models allow nonequilibrium melting and solidification to occur at temperatures other than the thermodynamic phase transformation temperatures and thus have tremendous flexibility in treating the many complicated phenomena that occur during pulsed laser annealing and ablation.

A previously developed 2-D LASER 8 model has been applied recently to study several problems during annealing and ablation of solid materials by pulsed laser beams $[3,4]$, including: (1) the dynamics of "explosively" propagating buried liquid layers during pulsed laser annealing of a-Si layer on C-Si substrate, (2) the dynamics of Si flake nucleation and growth and the final structures obtained with different laser energy densities, (3) ablation due to absorption by localized but extended defects such as in the case of $\mathrm{MgO}$, (4) particulate ejection during laser ablation and matrix assisted laser desorption ionization (MALDI). Fig.1 shows one example from our modeling and simulations of explosive crystallization. Quantitative and qualitative agreement achieved between experiment and theory demonstrates that the approach employed in the simulations can be generally applied to a variety of problems and for many other materials. The 2-D program has been rewritten for massively parallel machines such as the Intel Paragons in ORNL's Center for Computational Sciences by one of us (CLL), thus allowing larger and more accurate calculations for complex systems to be carried out in reasonable times.

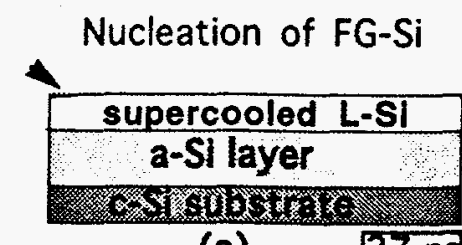

(a)

Propagation of flat BLL

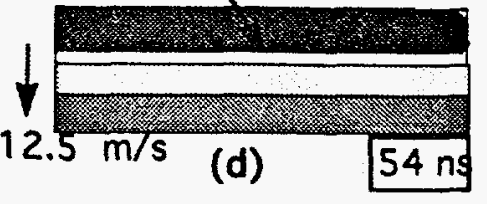

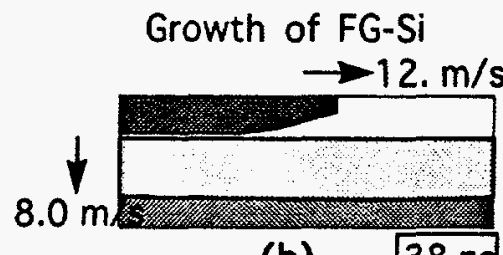

(b)
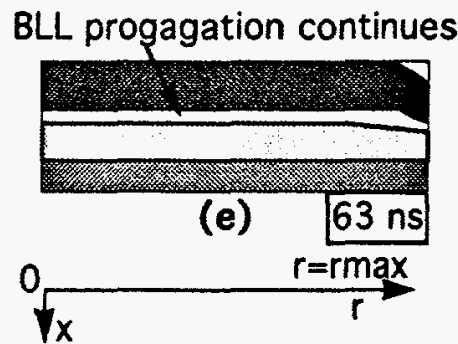

Formation of BLL

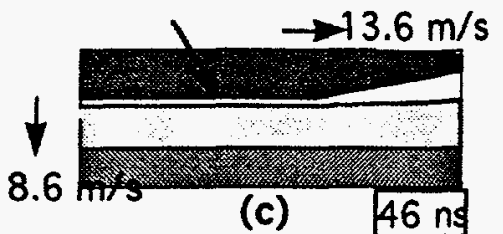

Flat FG-Si/a-Si interface

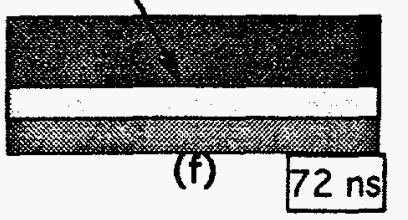

Fig.1 The nucleation and growth of a Si flake (fine grain Si, FG-Si), formation and propagation of the buried liquid layer (BLL), and calculated flake growth and BLL propagation velocities as a function of time. In this case, an amorphous $\mathrm{Si}$ (a-Si) layer $410.0 \mathrm{~nm}$ thick on a crystal Si (cSi) substrate was irradiated by a $\mathrm{KrF}$ laser pulse with full width at half maximum of $40.0 \mathrm{~ns}$ at an energy density of $0.25 \mathrm{~J} / \mathrm{cm}^{2}$. Simulations are done for half the flake (from $\mathrm{r}=0$ to $\mathrm{r}=\mathrm{rmax}$ ) because of symmetry.

\section{Vapor breakdown}

A simple kinetic model was developed to simulate the vapor breakdown during ablation of a Si target $[5,6]$. Two absorption mechanisms are considered for vapor heating through laser beam-plume interaction, i.e., inverse bremsstrahlung (IB) and photoionization of excited states. The rates of various processes were calculated according to the formulas given by Zel'dovich and Raizer [7]. The rate equations were solved for five quantities, namely, densities of electrons, neutrals, and excited states, and the temperatures of electrons and heavy-body particles (ions, neutrals, and excited states), much as was done earlier by Rosen et al [8]. Resupply of the vapor by evaporation and dilution of it due to expansion were also taken into account in the calculations. Only singly charged particles were considered and a $\mathrm{KrF}$ eximer 
laser pulse with full width at half maximum (FWHM) of 40.0 ns was used in the calculations. The total breakdown times (the sum of evaporation time and vapor breakdown time) at different energy fluences were then calculated. The results indicated that at an energy fluence of 6.0 $\mathrm{J} / \mathrm{cm}^{2}$ the total breakdown surprisingly occurred toward the end of the laser pulse at $46.0 \mathrm{~ns}$. In this case, one may not see the vapor breakdown visibly by means of light emission. On the other hand, at an energy fluence of $20.0 \mathrm{~J} / \mathrm{cm}^{2}$, one may readily see the breakdown in the time frame (the total breakdown time $-24.0 \mathrm{~ns}$ ) of the laser pulse duration. This is qualitatively consistent with our experimental observations of Si target ablation using a KrF laser.

\section{Gas dynamic modeling of plasma transport in vacuum and in background gas}

1-D and 2-D coupled gas dynamic equations describing the conservation of mass, momentum, and energy were solved numerically to simulate plasma expansion and transport both in vacuum and in background gas. The implementation of the $1-\mathrm{D}$ model $[5,6]$ is similar to that used by Vertes et al (1-D) $[9,10]$ except that the Rusanov scheme [11] is used to advance the quantities in the hydrodynamic equations as a function of time. The Rusanov scheme was chosen since it is capable of describing shock waves approximately if there are any during plasma expansion and transport. The 1-D model $[5,6]$ includes features such as absorption of laser beam energy through inverse bremsstrahlung (IB) and photoionization processes, an equation of state for an ideal gas and its internal energy density, and the Saha equation for ionization of the vapor. Dilute background gas is also introduced as a second entity in the 1-D model to simulate the hydrodynamic effects of interactions between the plume and the background on the evolving flow field of the plume [6]. Viscous and scattering effects have also been included. The main equations for the 2-D model used are:

Density:

$$
\frac{\partial}{\partial t} \rho=-\frac{\partial}{\partial x}\left(\rho v_{x}\right)-\frac{\partial}{\partial y}\left(\rho v_{y}\right)
$$

Momentum:

$$
\begin{aligned}
& \frac{\partial}{\partial t}\left(\rho v_{x}\right)=-\frac{\partial}{\partial x}\left(p+\rho v_{x}^{2}\right)-\frac{\partial}{\partial y}\left(\rho v_{x} v_{y}\right) \\
& \frac{\partial}{\partial t}\left(\rho v_{y}\right)=-\frac{\partial}{\partial x}\left(p+\rho v_{y}^{2}\right)-\frac{\partial}{\partial x}\left(\rho v_{x} v_{y}\right)
\end{aligned}
$$

Energy:

$$
\begin{aligned}
& E=\rho \varepsilon+\frac{1}{2} \rho\left(v_{x}^{2}+v_{y}^{2}\right) \\
& \frac{\partial}{\partial t} E=-\frac{\partial}{\partial x}\left[v_{x}(E+p)\right]-\frac{\partial}{\partial y}\left[v_{y}(E+p)\right]
\end{aligned}
$$

where $\rho$ is the local particle density, $p$ the local pressure, $v$ the local fluid velocity, $\varepsilon$ the local internal energy density.

One of the technical issues for PLD is the capability of attaining large area deposition of uniform thin films. Many approaches have been tried to improve uniformity. One of the ideas is to use two overlapping plumes as a way to obtain large area deposition and better uniformity. We have simulated expansion and transport of two interacting plumes using the 2-D hydrodynamic model and a typical output is shown in Fig.2. As can be seen, two plumes are initially generated near the target surface. As time goes by, both plumes expand util at a later time, they contact each other. A much smoother propagating front covering a large area develops as a result of the interaction. 


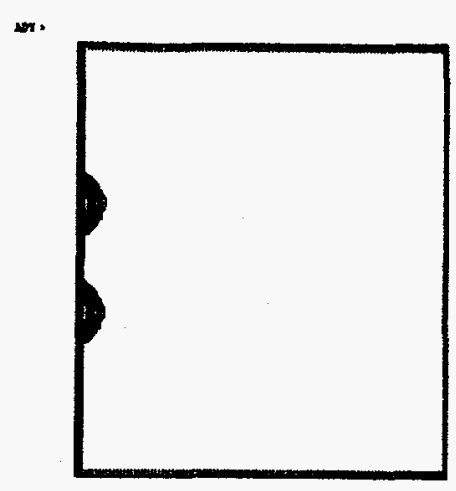

(a)

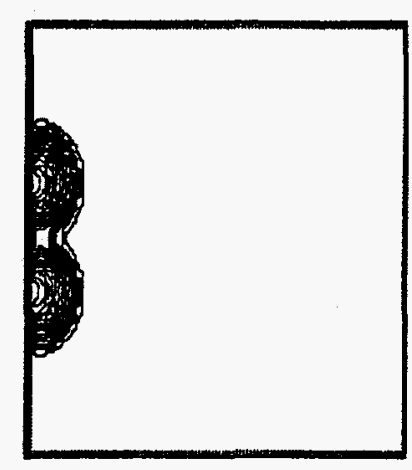

(b)

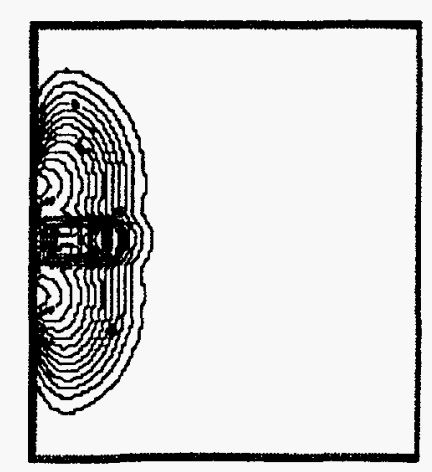

(c)

Fig.2 2-D gas dynamic modeling of two plume expansion for PLD. (a) starting condition (b) two plumes expand (c) two plumes interact and a smooth propagation front is developed.

The results obtained so far from gas dynamic modeling have shown that there are shock waves formed near the expanding front and shock heating and ionization are observed during plume expansion into background gas. It is also shown that the background gas is "snow plowed" by and mixed with the leading edge of the plume. The resulting increase in pressure at the propagating front results in a pressure gradient acting on the main body of the rarefied plume, tending to slow it down and in some cases even resulting in backward propagating materials.

\section{Particle hydrodynamic modeling of plasma transport both in vacuum and in background gas}

A 2-D particle hydrodynamic model has been developed by Leboeuf and collaborators $[12,13]$ to trace the position and velocity of individual particles in a plasma. Plasma expansion and transport were simulated by setting up different initial velocity distributions for the particles $[5,6]$. Background gas may play an important role for pulsed-laser deposition. We have carried out some simulations using the 2-D hydrodynamic particle model to simulate the interactions between the plume particles and the background particles [6]. The advantage of utilizing the particle model for such a case is that momentum transfer among the particles within a hydrodynamic cell is freely allowed. A dragging term from viscosity can be added to the driving force. A typical output is shown in Fig.3. The starting condition is such that all the particles in the plume have uniform initial flow velocity, but with background particles surrounding the plume and throughout the rest of the area. At an early time (b, in Fig.3), we see the plume particles are "snow plowed" into the background particles and two fronts, namely the expanding front and a contact front are clearly seen. A mixing zone exists between the two fronts. At a later time, some plume particles escaped from the mixing zone and thraveled through the back ground gas, as seen in Fig.3c.

\section{Dynamic deposition processes of energetic particles and thin film quality}

It has been found [14] that there are two components in the time of flight (TOF) signals: a fast-moving component that travels at an almost constant velocity through the dilute background gas, and a slow-moving component whose velocity depends on the background pressure. It is believed that the fast-traveling component is harmful to the film deposition since the particles in it have higher kinetic energies. In the case of deposition of $\mathrm{Cu}$ films, the fast-moving particles can have kinetic energies of $\sim 200.0 \mathrm{eV}$ while for the slow-moving particles the kinetic energies 
are $-100.0 \mathrm{eV}$ under normal conditions. In order to assess the effects of particle kinetic energies on the film quality by PLD from a $\mathrm{Cu}$ target, we have performed atomistic simulations using

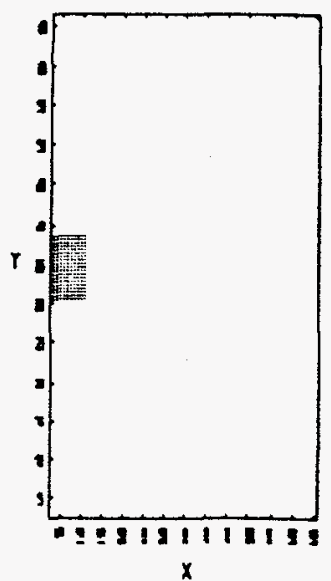

(a)

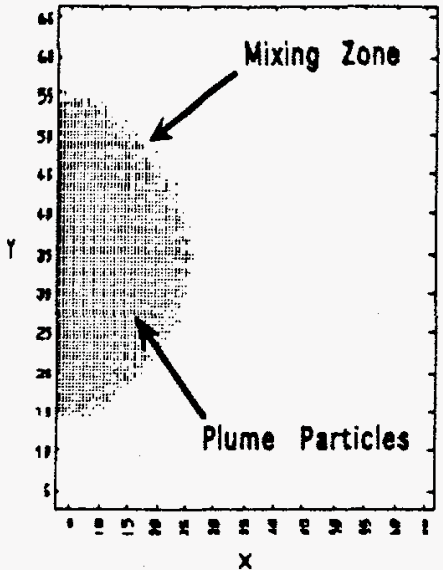

(b)

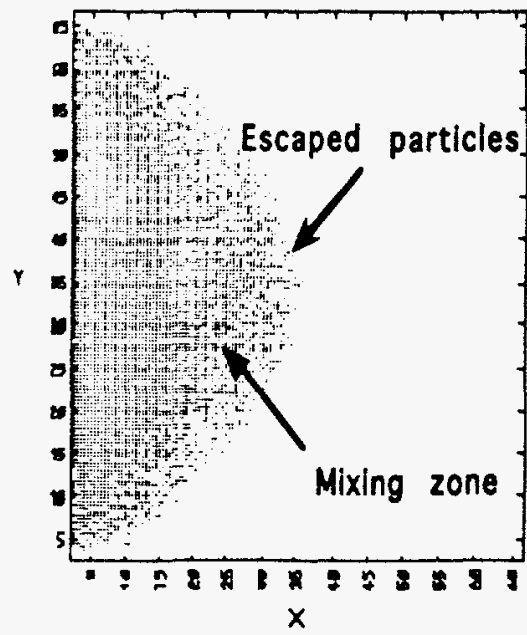

(c)

Fig.3 Particle-in-cell modeling of plume expansion in a background gas. (a) starting condition (b) development of mixing zone (c) escape of fast-moving particles from mixing zone.

molecular dynamics (MD) simulation techniques and the embedded atom method (EAM). A Cu particle above the $\mathrm{Cu}(100)$ surface is allowed to hit the surface at velocities corresponding to kinetic energies ranging from 100.0 to $200.0 \mathrm{eV}$. Trial work was done to identify the most probable point on the surface for the incoming particle to land; it was found to be the open channel along the $\langle 100\rangle$ direction on $\mathrm{Cu}(100)$. Trajectories of the particle and substrate atoms are recorded as a function of time. A critical kinetic energy for penetration of a $\mathrm{Cu}$ particle into $\mathrm{Cu}(100)$ surface is found to be $\sim 150.0 \mathrm{eV}$. As seen in Fig.4a, the energetic particle with a kinetic energy of $200.0 \mathrm{eV}$ can penetrate the first layer of $\mathrm{Cu}(100)$ surface. At the critical kinetic energy, the particle almost touches the surface atoms but does not penetrate. The trajectory of the particle with a kinetic energy of $150.0 \mathrm{eV}$ as seen in the plane perpendicular to the incident direction is given in Fig.4b. The hopping distances are large initially and gradually decrease as the particle loses its kinetic energy. It finally comes to rest after hopping about 5.0 $A$ along the $<110>$ surface channel.

Through the MD simulations, we see that differences in the kinetic energies of the plume particles by about $100.0 \mathrm{eV}$ do affect the impact behavior of the particles and, presumably, the resulting film quality. When the energetic particles impinge the substrate, it is expected that they promote surface dynamic processes such as surface diffusion. In this way, high quality thin films can be grown at a relatively low temperature. However, particle penetration is harmful for film growth in most cases since it can cause strains in the film and create some defects that can not be annealed out. The resulting unbalanced strains in the films often cause cracking.

\section{ACKNOWLEDGMENTS}

This research was sponsored by the Division of Materials Sciences, U.S. Department of Energy under Contract No. DE-AC05-84OR21400 with Martin Marietta Energy Systems, Inc., and in part by an appointment to the Oak Ridge National Laboratory Postdoctoral Research Associates 
Program administered jointly by the Oak Ridge National Laboratory and the Oak Ridge Institute for Science and Education.

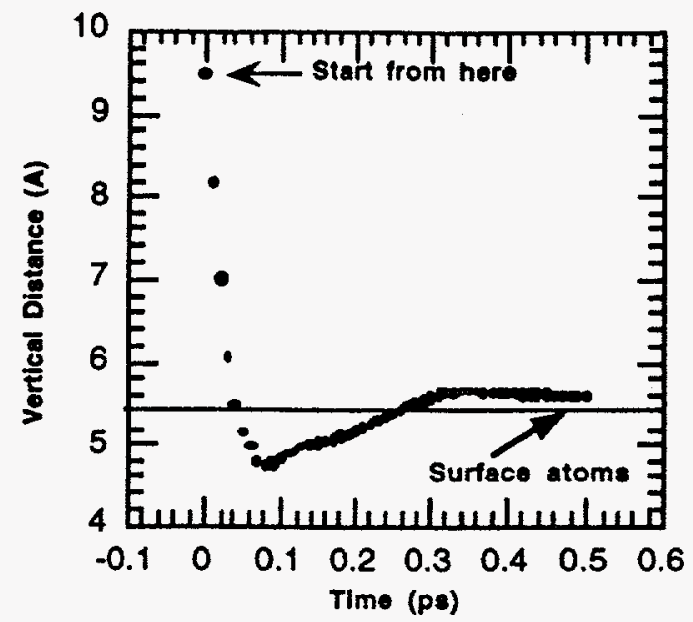

(a)

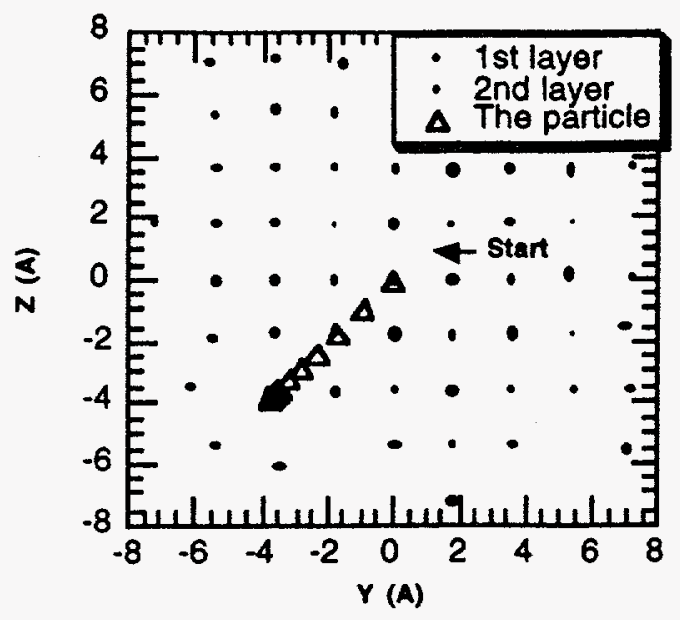

(b)

Fig.4 (a) Penetration of the particle with a kinetic energy of $200.0 \mathrm{eV}$. Time step for the MD simulations used is $1.10^{-15} \mathrm{~s}$. (b) Atomic view of the particle trajectory hopping in $\langle 110\rangle$.

\section{REFERENCES}

1. R. F. Wood and G. A. Geist, Phys. Rev. Lett. 57, 873 (1986).

2. R. F. Wood and G. A. Geist, Phys. Rev. B 34, 2606 (1986).

3. R. F. Wood, Al Geist, and C. L. Liu, submitted to Phys. Rev. B.

4. C. L. Liu, R. F. Wood, and D. H. Lowndes, to be submitted to Phys. Rev. B.

5. C. L. Liu, J. N. Leboeuf, R. F. Wood, D. B. Geohegan, J. M. Donato, K. R. Chen, and A. A. Puretzky, Proceedings of the International Conference on Processing and Advanced Applications of Lasers (ICPAAL), Palm Coast, Florida, May 1-6, 1994

6. C. L. Liu, J. N. Leboeuf, R. F. Wood, D. B. Geohegan, J. M. Donato, K. R. Chen, and A. A. Puretzky, Gordon Research Conferences, New London, New Hampshire, August 14-19, 1994.

7. Ya. B. Zel'dovich and Yu. P. Raizer, Physics of Shock Waves and High-Temperature Hydrodynamic Phenomena, Academic Press, New York and London, 1966.

8. D. I. Rosen, J. Mitteldorf, G. Kothandaraman, A. N. Pirri, and E. R. Pugh, J. Appl. Phys. 53, 3190 (1982).

9. A. Vertes, P. Juhasz, M. D. Wolf, and R. Gijbels, Scanning Microscopy 2, 1853, 1988.

10. L. Balazs, R. Gijbels, and A. Vertes, Analytical Chemistry 63, 314 (1991).

11. V. V. Rusanov, J. Comp. Math. Math. Phys. USSR, No. 2 (1962); G. A. Sod, Comput. Phys., 1(1978).

12. J. N. Leboeuf, T. Tajima, and J. M. Dawson, J. Comput. Phys. 31, 379 (1979).

13. F. Brunel, J. N. Leboeuf, T. Tajima, J. M. Dawson, M. Makino, and T. Kamimura, J. Comput. Phys. 43, 268 (1981).

14. D. B. Geohegan, Appl. Phys. Lett. 60, 2732 (1992). 